\title{
MACHINE WORK PLANNING USING WORKPIECE AGENTS IN AN AUTONOMOUS DECENTRALIZED MANUFACTURING SYSTEM
}

For High-mix Very-low Volume Production

\author{
Michiko Matsuda, Satoshi Utsumi and Yasuaki Ishikawa \\ Kanagawa Instistute of Technology, Atsugu-shi, Kanagawa, 243-0292 Japan; Email:, \\ matsuda@ic.kanagawa-it.ac.jp
}

\begin{abstract}
In this paper, a workpiece agent is proposed for the configuration of an autonomous decentralized manufacturing system which flexibly responds to high-mix, very-low volume production. When a blank workpiece is put on to the production line, the workpiece agent is dynamically generated with the product model. According to the state of each machine tool on the shop floor, the agent plans the machining processes by itself. Then the workpiece agent determines the selection of the machine tools for each machining step and obtains a schedule in real time through the negotiation among two or more workpiece agents aiming to increase efficiency of the whole manufacturing system. Trial implementation of a workpiece agent has been done.
\end{abstract}

Key words: multi agent system; workpiece agent; agent negotiation; machine work planning; event driven manufacturing system

\section{INTRODUCTION}

In recent years, the requirement for high-mix very-low-volume production including flexible order-made production is increasing. Constructing autonomous decentralized manufacturing systems is one of the solutions for the realization of high-mix very-low-volume production. In an autonomous decentralized manufacturing system, a manufacturing activity unit such as machine tool, robot $\mathrm{AGV}$, and/or manufacturing cell has autonomous functionalities. On the other hands, multi agent technology is recently being well applied to construct autonomous systems. The elements

Please use the following format when citing this chapter:

Matsuda, Michiko, Utsumi, Satoshi, Ishikawa, Yasuaki, 2006, in International Federation for Information Processing (IFIP), Volume 207, Knowledge Enterprise: Intelligent Strategies In Product Design, Manufacturing, and Management, eds. K. Wang, Kovacs G., Wozny M., Fang M., (Boston: Springer), pp. 869-874. 
in an autonomous system are configured as an agent. Multi agent technology is also applied to the constructions of the autonomous decentralized manufacturing system[1]. Manufacturing activity units, especially manufacturing devices are configured as agents. In these cases, system structure is a device oriented structure. To obtain more flexibility and autonomy, an event driven system structure is useful. For the manufacturing system, the occurrence of an event means that a workpiece is input to the manufacturing shop floor. This paper proposes to represent the workpiece as a dynamic agent. This agent is called a "workpiece agent."

\section{MANUFACTURING SYSTEM BASED ON THE WORKPIECE AGENT}

A workpiece agent is an intelligent and autonomous software unit attached to a workpiece. This workpiece agent plans the processes and allocates the manufacturing machine for executing the processes required to generate the product. This means that the workpiece agent takes an initiative in the manufacturing system. The conceptual structure of the autonomous decentralized manufacturing system based on a workpiece agent is shown in Figure 1. The workpiece agent is a dynamic agent. It is created when the workpiece is input to the shop floor. The workpiece agent is deleted when the product is completed. At first, after its creation, the workpiece agent generates a process plan. Then the workpiece agent negotiates with other workpiece agents which are already on the shop floor to obtain the manufacturing machine allocation, and orders an AGV for the workpiece transfer. Later on we will discuss the machining system based on workpiece agents.

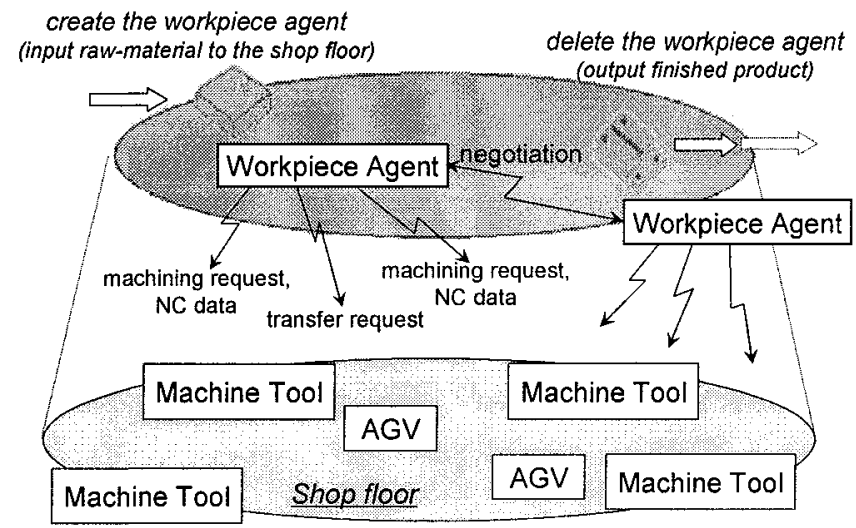

Figure 1. Concept of the workpiece agent based manufacturing system. 


\section{STRUCTURE OF THE WORKPIECE AGENT}

When a workpiece agent is created, it has machining knowledge and product data which describes the finished product. The structure of the workpiece agent is shown in Figure 2. The workpiece agent has a machine work planning function and a machining/transfer requesting function.

At first, several possible machining process plans are generated in the workpiece agent by referring to the product model, machining knowledge and machine tool data which models the available machine tools on the shop floor. Then, workpiece agent selects an executable plan by referring the current machining job allocation schedule, and negotiates with the other workpiece agents by requesting schedule changes according to the selected plan. After a successful negotiation the workpiece agent requests an AGV for a transfer and asks the machine tool for machining by verifying the machining job allocation schedule on the information board of the shop floor.

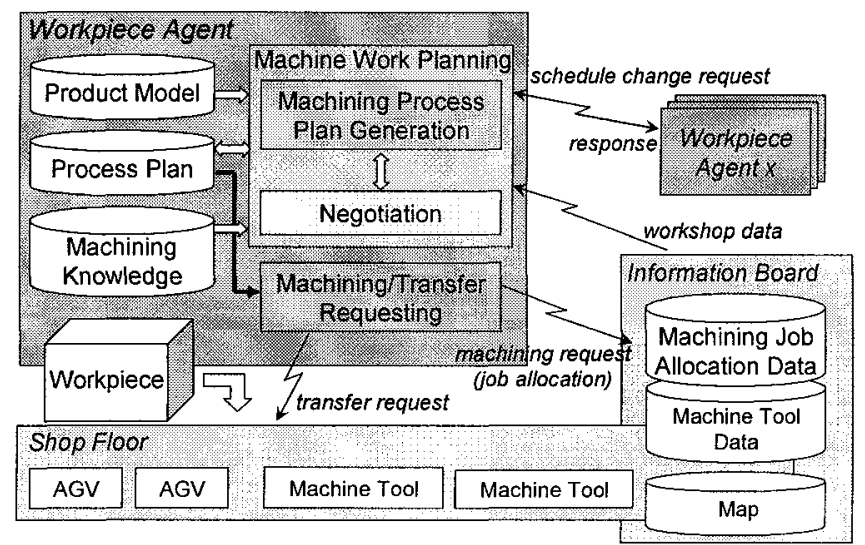

Figure 2. Structure of the workpiece agent.

\section{MACHINE WORK PLANNING}

\subsection{Machining process planning}

The first step in the machining process planning procedure is extracting machining features from the finished product model. The second step is lining up candidates of machining processes depending on features using the Feature-Machining Method Table. In this table, possible machining methods for producing corresponding features are described. Usually, one feature 
type has several possible machining methods. The contents of this table is machining knowledge. The third step is the narrowing down of candidates using machine tool data on the information board. Machining tool data shows available machine tools and machine tool status on the shop floor.

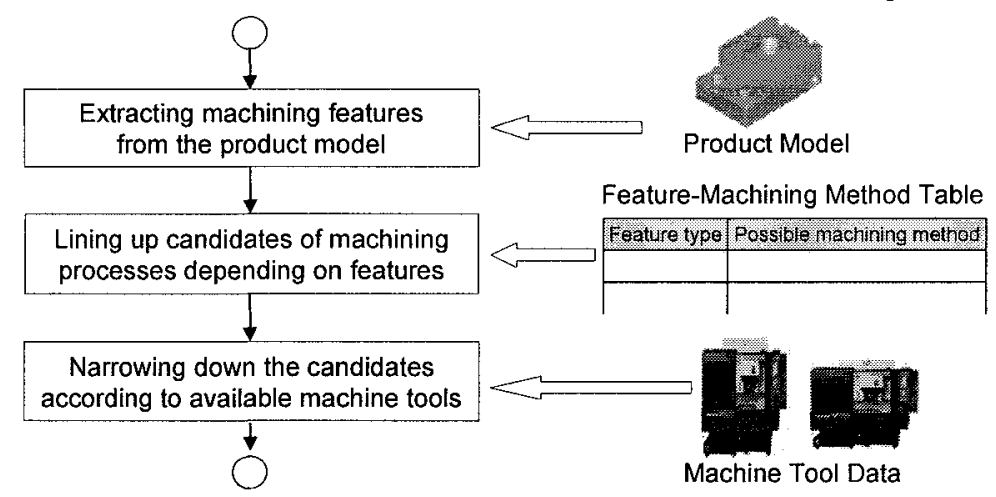

Figure 3. Machining process plan generation in the workpiece agent.

\subsection{Machine work scheduling by one-to-one negotiation}

When the workpiece is input to the machining shop floor with some urgency machine work scheduling is done by a one-to-one agent negotiation after process planning as shown in Figure 4. In Figure 4, Workpieces A, B and $\mathrm{D}$ are already on the shop floor, and Workpiece $\mathrm{D}$ is input with some urgency. Machine A and B have the same specification. It is recognized that Machine $B$ has a time margin by referring to the current schedule. Therefore the process plan for Workpiece D which Machine B is able to execute is selected and a candidate for am interrupt schedule is generated. According to this interrupt schedule, Agent $D$ negotiates with Agent $C$ to concede the Machine B allocation. If Agent $\mathrm{C}$ accepts this offer, Agent $\mathrm{C}$ refers the new current schedule and negotiates with Agent B. Agent $B$ then repeats as same.

\subsection{Machine work scheduling by one-to-many negotiation}

One-to-one agent negotiation provides a locally optimized machine work plan. To get totally optimized machine work plan, one-to-many agent negotiation is proposed. Figure 5 shows the scheduling process for one-tomany agent negotiation. The case of Figure 5 is the same as in Figure 4. Product D is input to the shop floor. Before the negotiation, an optimized schedule is generated by applying the genetic algorism[2]. One individual 
Decentralized Manufacturing System

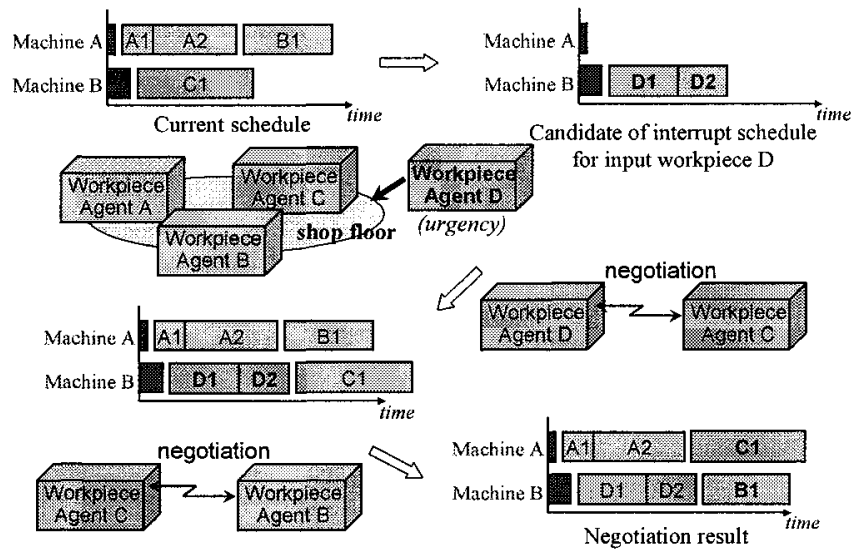

Figure 4. Machine work scheduling by one-to-one negotiation.

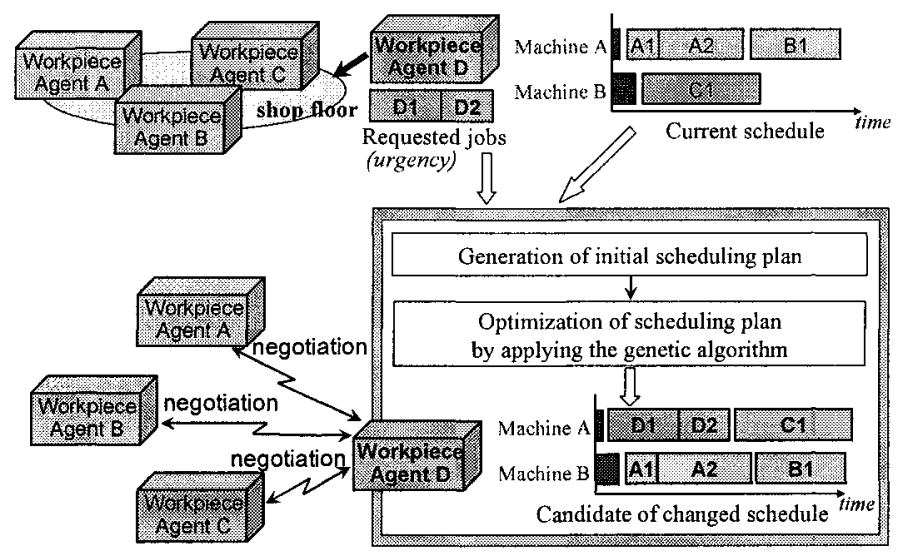

Figure 5. Machine work scheduling by one-to-many negotiation.

represents one scheduling plan. Initial individuals are generated by setting required processes for Workpiece $\mathrm{D}$ into the current schedule according to a setting rule. Then, genetic procedure is applied to the initial individuals. The most optimized schedule is the first candidate for the changed schedule. According to this schedule, Agent $\mathrm{D}$ negotiates with Agent $\mathrm{A}, \mathrm{B}$ and $\mathrm{C}$ at same time. If negotiation is not successful, another processes for Workpiece $\mathrm{D}$ is selected and the procedure is repeated.

\subsection{Planning simulation using workpiece agents}

A Planning Simulation using workpiece agents is now performed. Figure 6 shows the result of the example simulation. There are three types of 
products: A, B and C. A product of type D interrupts the line. There are four milling machines on the shop floor. The specifications for the milling machines are different. A1-1 represents product type, lot number and process number. In Figure 6, the upper chart is the result for the case of one-to-one negotiation and the lower chart is for the case of one-to-many negotiation. One-to-many negotiation provides a more even and efficient schedule.

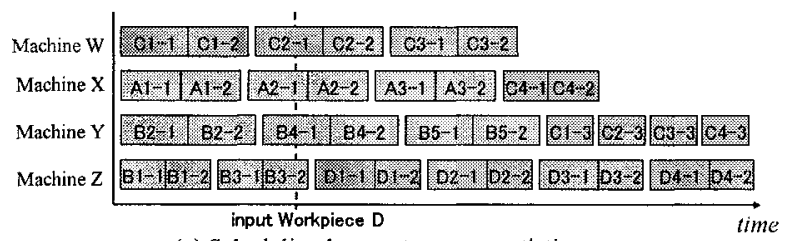

(a) Scheduling by one-to-one negotiation

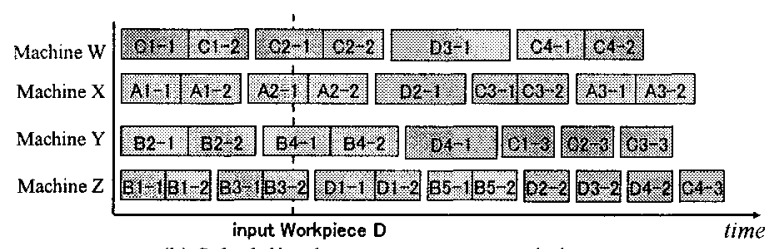

(b) Scheduling by one-to-many negotiation

Figure 6. Example results of machine work planning simulation.

\section{CONCLUSIONS}

Workpiece agents and the configuration of autonomous machining systems using workpiece agents are discussed in this paper. The concepts of a workpiece agent is that a workpiece has the initiative in the manufacturing process and could autonomously decide on the manufacturing process and the allocation of manufacturing machines for itself. Trial implementation of the workpiece agents and simulation of machine work planning using workpiece agents show the effectiveness of event driven configurations on the manufacturing system.

\section{REFERENCES}

1. N. Sugimura, R. Shrestha, J.Inoue, Integrated process planning and scheduling in holonic manufacturing systems -Optimization based on shop time and machining cost-, Proc. of the 2003 IEEE International symposium on Assembly and task planning (ISATP2003, 2003), pp. 36-41.

2. Y. Tanimizu, T. Sakaguchi, K. Harada, N. Sugimura, Genetic Algorithm Based Reactive Scheduling in Multi-layered Manufacturing System, Proc. of 2002 Japan-USA Symposium on Flexible Automation, 2, (2002), pp.951-958. 